\title{
The status quo and prospect of the gastrointestinal cancer immunotherapy
}

\author{
Zhiqiang JIANG \\ Henan Tumor Hospital \\ Zhengzhou,Henan,China
}

\begin{abstract}
Modern tumor has entered the stage of elucidating the pathogenesis from the molecular level and to explore of convenient and effective treatment for early diagnosis and the treatment of molecular biology. Gastrointestinal cancer is the most common cancer in the country. It is still ranked the first place in various malignant tumors which has the hardness of early diagnosis and treatment with great dangers. This paper studies in the inner relationship between Hsp70 and gastric cancer and colorectal cancer by immunohistochemistry and in situ hybridization technique for protein separation techniques. The study aims to explore the role of Hsp70 in cancer and colorectal cancer biological behavior which is in seeking the target substance of effective diagnosis and treatment.
\end{abstract}

\section{Keywords- Cancer; the status quo; treatment}

\section{INTRODUCTION}

1986, when Srivastava 3) and other people is seeking TSA in MCA-induced mouse, sarcomas isolated a molecular weight of 96000Da (gp96) glycoprotein. After using it in the immunized mice, they found that mice having repulsive force against tumors $\mathrm{gP} \%$ Source and the transplanted tumor cannot grow. Meanwhile, Ulrich found that the protein isoform of molecular 84000Da and 86000Da (P84 / 86) is in the same tumor. Subsequently it detected that gp96 and P84186 are homologous which are all belong to the same family of Hsps except that P84186 located in the cytoplasm, and gp96 is located in the endoplasmic reticulum. The same method confirmed Grp78 (Bip) is the homologous proteins of endoplasmic reticulum of Hsp70 in cytosolic. Further research found that using $\mathrm{gP} \%$ sarcoma extracted from the 6138 in immunized mice can stimulate the generation of 6138 sarcoma with specific cytotoxic T lymphocyte function (CTL).And then put it into 6138 and living cells cannot grow, so the mice received anti- tumor capabilities. As the same, they putted immunized Hsp70 on the BALB / c mice revealed that the mouse has the ability to repel sarcoma derived from Hsp70. When using other Hsps such as Hsp65, Hsp28 immunized mice; they found they also have the tumor immunogenicity in ras transgenic tumor cells. Hsps induce a strong immune specificity MethA sarcoma gP\% for MethA immunogenic while there is zero with CMSS, and vice versa. There is happened with Gp96 of CMSS and CMS5 while MethA is not. MethA sarcoma Hsp90 and Hsp70 have the same features. Studying in BALB / c three sarcoma (MethA, CMSSCMS13) found that CMSS in mice is to produce antigp96 MethA or CMS13. Gp96 of MethA has specific immune cells for MethA role while it with CMS13 is some cross-immunity, but has no effect on the CMSS. The gp96 of CMS13 in the mouse is possible to make the ability to generate the abilities of exclusion with CMS13and partial exclusion with MethA, but have no effect on the CMSS. Palladin think that there may be have some cross antigen between CMS13 and MethA.

\section{HSPS ACCOMPANIED ANTIGEN BELLY}

In general speaking, that the body produces its own antigens is the result of genetic mutation. The $\mathrm{gP} \%$ and Hsp70 spleen cells of Normal BALB / c mouse are no immune function for MethA sarcoma. This seems that the immune function of Hsps is the result of the process of cell transformation gene mutation. But the sequencing in BALB / c spleen cells, MethA and the gp96cDNA gene of CMSS showed no polymorphism (c)\}.It means that there is no mutation. sequencing in other Hsps found the same results which you can exclude that Hsps immunogenicity is Hsps gene mutation . so someone speculated that Hsps molecular role of $\mathrm{N}$-linked polysaccharide (does not exist. a linked polysaccharide).However, the following experiments do not support this view: (1) MethA cells were cultured in medium containing Tunicamycine inspire BALB / c mouse MethA cells on specific repulsion. (2) MethA of gp96 in Tunicamycine medium cannot bind to conA-Sepharase, but they also have the ability of immunization. Thus they further speculated that there has mutation in the modification process of the protein after Hsps translation.

There has no polymorphism in gp96 gene itself. And $\mathrm{gP} \%$ is homologous in different sources. Gp96 stained gel showed a band on the silver. Using Edman degradation can find that there is only one amino terminus Hsps. C3 polypeptide using anti-gp96 antibody treatment can eliminate the immune activity. Then surely Hsps combine a variety of small molecular weight molecules such as polypeptides oHsps own immune molecules are not active but to transfer of antigens from the skin effect. Considering $\mathrm{gP} \%$ is located in the ER, they further speculated $\mathrm{gP} \%$ from polypeptide transfer receptor polypeptide which will cause ER, making it more accessible to MHCI molecules. In order to confirm whether the polypeptide binding with gp96, the $3 \mathrm{mg}$ Purified $\mathrm{gP} \%$ is be acid $(\mathrm{PH}=2)$, the use of HPLC chromatograph detected some low-molecular substances are eluted. And then collecting the substance under a specific absorption peak and analyzed by the mass spectrometer in SDS-PAGE detected macromolecular structural integrity, and then eluting no polypeptide absorption peak ' 7 ' which 
described that $\mathrm{gP} \%$ indeed binding with polypeptides sky polypeptide receptor (partner) in the ER. Further studies showed that Hsps can not only bind with polypeptides from the intracellular any protein degradation but polypeptide of immunogenic tumors. In the model of influenza virus, eluted polypeptide of influenza cells can make influenza-infected cells dissolved influenza-specific CTLs "'. Similarly, Hsp70 can also bind to tumor antigen peptide. The connected between Hsp70 and skin are sensitive to ATP. Hsp70peptide complex was added to make it free from ATP skin. if Hsp70- peptide complex purified by ATP-agarose column .gp96 no immunogenic polypeptides participate in the binding and separation of ER, and capable of binding or hydrolysis and ATP as an energy source, found $\mathrm{gP} \% \mathrm{~A}\} \mathrm{B}$ containing sequence homologous to two ATP-binding protein, but also, demonstrated in vitro gp96 can bind ATP, and ATP in the tag found gp96 cells, ADP binding. highly purified $\mathrm{gP} \%$ containing ATP activity, this activity can be monoclonal destruction, activity-dependent exogenous Mgz + but not $\mathrm{Ca} 2+$, gp96ATP activity optimum conditions for $\mathrm{PH}=7.2$, ATP activity in the multi-skin cannot be activated, and phosphorylated casein have this effect.

\section{SPECIFIC ACTIVE IMMUNOTHERAPY OF TUMORS}

Specific active immunotherapy of cancer mainly refers to the use of cancer vaccines stimulate the body to produce an immune response against the tumor-specific antigens which can be seen as one surgery for cancer patients, an adjuvant therapy with chemotherapy or radiotherapy. It aims at overcoming the immune suppression caused by the product tumor status and enhancing the immunogenicity of tumorassociated antigens to stimulate specific immune cells for attacking the tumor.There are several tumor vaccine with more current research such as tumor cell vaccines, vaccine antigens, vaccine based on dendritic fine (Dendriticcells, DC) and DNA vaccines, etc., but most current tumor vaccine research is still at the period of laboratory or clinical studies. There is little the practical application.

\section{A. Advantages of tumor cell vaccine tumor cell vaccine}

All molecules on cells including some unknown molecule can be exposed to the immune system. The body may generate an immune response against a plurality of molecules. However, there are several shortcomings in the presence of specific whole-cell vaccine such as poor immunogenicity, complex preparation and others, and its antigen complex components. So the effect is not obvious. The exogenous gene can be introduced into the tumor cells by adenovirus, retrovirus and other vectors. The object of modified cells vaccine is to enhance the immunogenicity of the tumor and enhance the reactivity of $\mathrm{T}$ cell to tumor antigens (such as the costimulatory Gene molecules transfected into tumor cells, providing a second signal of $\mathrm{T}$ cell activation) to stimulate anti-tumor immune responses. Some expression productions of the foreign gene may act directly on immune cells to promote the proliferation and differentiation of immune cells, thereby enhancing the body's anti-tumor effect. The most studied subjects are various cytokines and chemokines gene-modified tumor cell vaccine, various cytokines such as IL 2,4,6,12,18, GM CSF, etc. which are all introduced into a variety of tissue types and have different immunogenicity of tumor cells. There is a certain anti-tumor effect, but in the study of different tumor model, the effect is very different. The results of efficacy in clinical trials are not significant. How to improve the efficiency of gene vectors and security? How to efficiently target gene expression in specific target cells? Those are problems needed to be studied and solved.

\section{B. tumor antigen vaccine tumor antigens including tumor}

Specific antigens (TSA), tumor-associated antigens (TAA) and the tumor are seeing tumor antigen vaccine antigens (including TSA, TAA, as CEA carcinoembryonic antigen and prostate specific antigen PSA, etc.) as vaccines to stimulate the body to produce immune response against tumor cells. The complete gene are introduced into encoding antigen of a viral vector which was used to immunize patients. Tumor antigen vaccine containing multiple epitopes, Th cell epitopes including, CTL epitopes, B cell epitopes, etc...So it has a strong immunogenicity. What's more, it may induce tumor-specific CTL and antibody production to kill the target cells and inhibition of tumor growth. the preparation of peptide vaccine be isolated and purified from tumor membrane fractions and synthetic peptides (including epitope) and other methods to enhance the immunogenicity of TAA. Which has already achieved a certain effect in animal experiments and clinical. Studies on protein peptide vaccines are large .At the present, mucin core peptide, a mutant ras protein, P53 protein, Her 2 / neu protein, CEA, telomerase, heat shock proteins are seen as immunogens [13]. Peptide vaccine preparation preferably tumor-specific antigens (TSA) polypeptides, peptides such as MAGE 1 and 9 HLA A1 + antigen presenting cells (APC) in combination can be induced strong CTL responses [14].The breakthrough in this respect depends on the new identification and discovery of tumor-specific antigen.

DNA vaccine, also known as gene vaccines or DNA vaccines, is a gene encoding a specific antigen fragment into the eukaryotic expression vector. And then the plasmid DNA goes into a living body and other means by gene gun injection, intramuscular injection, liposome injected. So an exogenous gene expression in vivo is sustained. Antigen produced can activate the immune system. Plasmid DNA vaccine is stable, easy to pure and can be induced response against a particular epitome of the immune. The transfected host cells are capable of stably expressing an antigen which can meet the need to repeat the treatment. Although experimental studies have confirmed the feasibility in the nucleic acid in cancer treatment vaccine, due to technical limitations which includes how to make targeted gene and on what way can be efficiently expressed in a certain part in the multi-gene vaccine. The vaccine genes are currently still in the experimental stage and isn't into the clinical applications.

\section{DC-based tumor vaccine dendritic cells}

(DC) is the body's most powerful antigen-presenting cells (APC) and the first one initiates the immune response. Cellular immune response system activated by DC plays a 
leading role in the body's anti-tumor. The number and functions of DC have a closely relationship with the occurrence, development and prognosis of cancer. DC-based cancer immunotherapies are mainly in two ways: one is the DC vaccine, namely the impact of tumor antigen in vitro to DC, and reinfusion of tumor-bearing body; the other is a DCbased gene therapy, namely tumor antigen gene, cytokine gene or chemokine gene transfected DC reinfusion body. DC-based vaccines in clinical trials achieved some encouraging results. The tumors involved include B-cell lymphoma, prostate cancer, melanoma, multiple myeloma, renal cell cancer, lung cancer, colorectal cancer, breast cancer, and pancreatic cancer and non-Hodgkin s lymphoma. Currently, DC vaccine is the most studied and widely carry out in the clinical application of cancer vaccines. under the leadership of Professor Cao Xuetao, the Immunology Institute of Zhejiang University has done a lot of meaningful work in this regard at the past 10 years.

The aims of tumor-specific antigen stimulation DC are to transfer the antigens of tumor cell to DC. So that DC has tumor-specific and provide the necessary activation of $\mathrm{T}$ cell costimulatory signals which greatly enhance tumor immunogenicity. After using the tumor antigens in vitro pulsed DC, reinfusion in vivo or inoculated into tumorbearing organism which can induce antigen-specific CTL responses, generate a protective immune response and treat the tumor-bearing animal models which have been established. Some tumors seedlings have entered clinical trials. Due to ease of access and preparation of tumor antigens which tumor antigen-pulsed DC is commonly used in clinical methods , used in treatment and research of kidney cancer, malignant melanoma, prostate cancer and malignant glioma, and so on . But deficiencies mainly due to: nonassociated antigens and many kinds of large, easy to induce autoimmune disease; Antigenic peptides may not be used to induce the best anti-tumor immune response; and so difficult to determine the dose stimulation. DC fused with tumor cells formed / DC vaccine which derived from its capable of expressing the characteristic of antigens. Thereby it effectively enhances the tumor antigen-presenting ability handed host $\mathrm{T}$ cell recognition, to break immune tolerance. Which can produce significantly stimulated in vitro $\mathrm{T}$ cell proliferation and cytotoxicity specific CTL .And it can kill parental tumor in metastatic renal cell carcinoma and melanoma clinical / trials? It is better security, has achieved a certain effect. Genetically modified DC vaccine is tumor antigen gene or cytokine gene transfected DC. This can make an antigen molecule or cytokine expression in longterm stability within the DC. Therefore it has a better effect to stimulus. Although this method is not yet mature and there are little prepared DC vaccines in clinical studies, but still the hotspot of DC vaccines. A number of studies have shown that genetically modified DC vaccine has a good effect for lung cancer, prostate cancer, nasopharyngeal cancer, malignant melanoma and other tumors.

\section{D. subcellular structures vaccine}

Subcellular structures vaccine is membranous vesicle secreted by cells into the extracellular. Its surface contains a lot of protein and lipid components which are closely related with functions and sources. Such as dendritic cells and exosome secreted from tumor cells can simultaneously express MHC, class of molecules, costimulatory molecules, heat shock protein HSP70 90 and tumor antigens which can effectively assist antigen presentation and break the existing immune tolerance. In vivo and vitro, it can induce and enhance the body's immune response show the similar antigen-presenting cell function. As a subcellular component, exosome has some advantages of clear, stable activity, safe, effective and convenient to store in the treatment of tumors which shows good potential applications. Using the antigenpresenting function of exosome can providing a new strategy for cancer immunotherapy in prevention which has shown some results, such as in patients with advanced melanoma and lung cancer in exosome-based immunotherapy which have been observed clear tumor reversal . But exosomebased cancer immunotherapy still have many problems to be solved in principle. And there are many things unclear such as the operating method of preparing exosome optimized and the patient the best injection intervals, dose, adjuvant and joint applications.

\section{CONCLUSIONS}

The combination of a variety of immune therapies as well as joint with the other three treatments will reflect its superiority. On the basis of further understanding with escape mechanisms of tumor immune and the tumor microenvironment of tumor immunotherapy will become a more rapid progression of the emerging field of research and one of the important measures for comprehensive cancer treatment.

\section{References}

[1] Hong Jin, Chen Xiaosong, Shen Wei Kun < The Status and Prospect of breast cancer immunotherapy $>$ [J] tumor, 2013,12: 1125-1129.

[2] Jiaoshun Chang<The status quo of tumor immunotherapy> [J] Chinese Journal of New Drugs, 2014,17: 2039-2042.

[3] Zhang Lihuang, Wang Qingqing<The Present and Future in cancer immunotherapy $>[\mathrm{J}]$ Zhejiang University (Medical Sciences), 2010,04: 339-344.

[4] Zhang xiaoren, Gehai Liang, Zhou guang Yan<The status quo and challenges of tumor immunotherapy $>[\mathrm{J}]$ Chinese Journal of Cancer Biotherapy, 1997,03: 189.

[5] Ren jun <The status quo and prospect of cancer immunotherapy> [J] Capital University of Medical Sciences, 2012,05: 597-601.

[6] Yeyun Bin, Zhou Zhifeng <The Status and Prospect of primary liver cancer immunotherapy> [J] Chinese Journal of Cancer, 2011,02: 108 114. 